\title{
Free Form CMOS Electronics: Physically Flexible and Stretchable
}

\author{
M.M. Hussain*, J.P. Rojas, G.A. Torres Sevilla, A.M. Hussain, M.T. Ghoneim, A.N. Hanna, A.T. Kutbee, J.M. Nassar and M. Cruz \\ Integrated Nanotechnology Lab, Electrical Engineering, Computer Electrical Mathematical Science and Engineering Division \\ King Abdullah University of Science and Technology (KAUST), Thuwal 23955-6900, Saudi Arabia \\ *Corresponding author’s coordinates: +966-544-700-072 and muhammadmustafa.hussain@kaust.edu.sa
}

\begin{abstract}
Free form (physically flexible and stretchable) electronics can be used for applications which are unexplored today due to the rigid and brittle nature of the state-of-the-art electronics. Therefore, we show integration strategy to rationally design materials, processes and devices to transform advanced complementary metal oxide semiconductor (CMOS) electronics into flexible and stretchable one while retaining their high performance, energy efficiency, ultra-large-scale-integration (ULSI) density, reliability and performance over cost benefit to expand its applications for wearable, implantable and Internet-of-Everything electronics.
\end{abstract}

Introduction: Over the years CMOS electronics have expanded from the field of computation to communication, entertainment and automobiles. Looking forward, serious opportunities exist in the general areas of advanced and low-cost healthcare, stylish product design, human machine interfaces, environmental sensing and monitoring, security and interactive world of information and electronics. Still to expand in these areas, it is imperative to develop electronics which are physically flexible and stretchable to be compliant to conform on various asymmetric surfaces related to living beings (human, animals, plants) and biological systems (both in-vivo and in-vitro). Such intimate conformity is critical for sensing and actuation.

Status-quo: We are already observing high interest in flexible macroelectronics such as large area display, energy harvesting and storage - where organic, molecular, nano-particles, nanowires/tubes and 2D materials (such as graphene, dichalcogenides) can be instrumental due to their low-cost processing [1, 2]. However, for data relevant flexible and stretchable electronic systems where in-situ data generation, their processing, storage, transmission is necessary, hybrid integration of CMOS electronics with heterogeneous materials is critical. For simple system, $\mathrm{mm}^{2}$ scale integrated chip (IC) may suffice but multi-tasking high performance system requires $\mathrm{cm}^{2}$ processors which necessitates their flexing and stretching too. Existing options for flexible and stretchable CMOS electronics include but not limited to: (i) back grinding which is abrasive and thus limits thickness reduction for enhanced flexibility; (ii) ultra-thin flexible silicon - large defect densities limit their usage for high quality electronics and they are severely bowed after a few high thermal budget processes; (iii) highly expensive substrate with buffer layers such as silicon-on-insulator (SOI) can be used by removing the buffer layer (i.e. buried oxide layer) to obtain flexible and ultra/extreme thin (SOI) electronics [3-5]. In the past, various techniques (Figs. $1-3$ ) have been demonstrated to make flexible CMOS electronics: (i) microwires/devices formation from SOI or unorthodox Si (111) substrates and their transfer printing - cost and integration density are two major areas of improvement; (ii) formation of porosity in the substrates using expensive processes followed by low-throughput epitaxial film growth prior to device fabrication and then controlled pick and place; and (iii) spalling to spall ultra-thin layer of crystalline film with pre-fabricated devices using a stressor layer resulting into an overall expensive and complex process with risk of losing free-hanging microelectromechanical systems (MEMS) devices.

\section{Our approaches (experimental, results and discussions):}

To effectively preserve the essential attributes in the state-of-the-art electronics while making them flexible we have developed two CMOS compatible processes: (a) deterministic pattern based flexing-peel-reuse (Fig. 4) and (b) non-abrasive subtractive etch back based flexing (Fig. 5). In the first method we first build the devices following standard CMOS compatible processes. Next we identify the shallow trench isolation (STI) areas (where no active device is present). Most often, with careful mask design and device positioning, vertical alignment between of the STI areas and cross-linking multi-level interconnects can be avoided. Next using soft polymeric or hard thin film mask, we form a deterministic pattern of porous network (micro-air vertical channels) from top level of the multi-level interconnect, through STI oxides until we reach the silicon surface. We continue the reactive ion etching (RIE) down to silicon substrate to our desired depth. Then we form a vertical sidewall (spacers) around the micro-air vertical channels using various dielectric thin films. Typically a trade-off exists between the thermal budget of the thin film deposition and the density of the film. It is important to use the films whose deposition does not need higher thermal budget and at the same time they are selective enough to subsequent isotropic etch process. When the spacers are formed, then we flow xenon difluoride $\left(\mathrm{XeF}_{2}\right)$ isotropic etchant gas which etches the silicon in the vertically downward direction much faster than the lateral direction. Anti-gravity vertically upward direction etch rate is the slowest. Still, during the etch process we often form a series of scallops and interconnected cavities which allows the detachment of the top portion of the substrate with pre-fabricated devices. Such released (detached) substrate "fabric" are thin down to $5 \mu \mathrm{m}$. The thinner they are, the more flexible they are. Yet, they are fragile too. Presently we are using soft vacuum based mechanical robotic arrangement to detach and transfer the released fabric to the destination site. When the transfer is completed, we use laser scribing to dice the fabric. We have 
been successful to dice the 4 " diameter thin film fabric into 1 $\mathrm{mm} \times 1 \mathrm{~mm}$ small pieces. It is important to note that as we are discussing many new materials (like $2 \mathrm{D}$ atomic crystal structure materials) and transfer printing processes, destination site will play important role. Such destination substrate (site) needs to be thermally highly conductive but electrically insulating. Also, it might be effective to have destination site which is reversibly bi-stable in its mechanical state: rigid and flexible. Presently we are exploring options how we can functionalize various destination sites to serve these purposes. When the dices are ready to be distributed among various destination sites, next important process module is to connect them. Asymmetric arrangement (with at the least $5 \mu \mathrm{m}$ thick fabrics) of the device plane and the destination site, interconnect becomes complicated. Additionally, mechanical integrity of interconnect needs to be preserved during various mechanical deformation including flexing and stretching. Finally it is also important to develop robust but flexible packaging system to house all the finished systems.

While our first method allows to build flexible devices from the scratch, the second method is suitable when any off the shelf ICs can be etched back to cause material loss but thinning them down to $5 \mu \mathrm{m}$ or below. Although back grinding is the industry standard for such thinning, their abrasive mechanical nature limits their thinning down to $30 \mu \mathrm{m}$ only. This not only reduces the flexibility but also can cause severe damage of the existing devices. Therefore, we have developed our second method based on reactive ion etching in sequential manner and lasing based thinning process. Critical challenge exists with both of these processes is rougher back surface. This can sometimes facilitate better adhesion on final destination sites which are flat and smooth.

To obtain stretchable electronics, we have combined pre-strained naturally stretchable soft-materials with fractal design based rigid thin film based mono-crystalline electronics (Fig. 6). Extensive finite element method (FEM) analysis allows us to adopt proper geometric configuration to develop architecture which can absorb deformation strain. Such design enhances the mechanical lifetime of the fabricated devices and systems. Again, rational design of materials and processes is the key to obtain highly stretchable platforms.

Using both of our processes we have successfully demonstrated range of devices with flexibility down to $0.5 \mathrm{~mm}$ bending radius and $1000 \times$ stretching in silicon (100): high-k/metal gate based advanced MOS field effect transistors (both planar and non-planar FinFETs) for logic applications (Figs. 7, 8) [13, 14], metal-insulator-metal capacitors (MIMCAPs) (Fig. 9) [15], ferroelectric and memristor for memory applications [16, 17], thin film transistors (TFTs) (Fig. 10), thermoelectric generators (Fig. 11) [18] micro lithium ion battery ( $\mu$ LIB) (Fig. 12), antenna (Fig. 13), sensors and actuators (including a smart thermal patch for pain management) (Fig. 14) [19]. In all devices, we have carried out extensive electrical, mechanical, biological functionality and reliability characterizations especially under various mechanical deformation conditions including bending (compressive and tensile) at various radiuses, twisting at various angles, stretching and contracting (Fig. 13 17). In all cases we observe that the device functionality and reliability can be impaired under extreme mechanical conditions $[20,21]$. That necessitates careful and rational design of materials and devices to make the overall system physically unyielding and electronically efficient. Finally the processes have been expanded for SiGe, Ge, and III-V based material systems including InP, GaAs, etc. [22, 23].

While free form electronics can expand the horizon of applications for electronics, an ongoing debate related to choice of material still sustains. It is obvious that self-assembled 0D, 1D and 2D devices offer flexibility and ruggedness however for data relevant systems their role can be significantly reduced to sensing and actuating purpose. One can argue that CMOS chips are small enough eliminating need to thin them down. In reality, the data management in each of these free form systems will be large enough which will require more transistors per unit area. Even with severe scaling down to $2 \mathrm{~nm}$ technology node, we can expect increased number of transistors and consequently increased real estate usage per chip. As an example, to host $4.5 \mathrm{~B}$ transistors, the real estate consumption will be $5.5 \mathrm{~cm}^{2}$. Such large platform, discrete chips need to be interconnected properly on the flexible destination platform to provide the data management backbone to other electronics made with $0 \mathrm{D}, 1 \mathrm{D}$ and 2D materials. Obviously if we can build all our electronics (including data management) with any of these materials, that would be the most optimal solution. However, many practical concerns still remain at large to promote the cause of transforming today's state-of-the-art electronics into flexible and stretchable one for their immediate use in the form of free form high quality electronics.

As we see surge of many new applications based on free form electronics, their high volume manufacturability is still uncertain. Major reasons include but not limited to use of unorthodox process techniques, thermal budget restriction and lithographic resolution limitation. Therefore, in our group we are focused to gradually establish start-to-finish process modules to provide mechanically robust and electrically high performing high quality free form electronics at an affordable price.

Conclusion: We have shown integration strategies to transform CMOS electronics into flexible and stretchable electronics. Future works include in-plane and out-of-plane transfer printing, aligning, wiring, bonding and packaging to achieve fully flexible and stretchable ICs and electronic systems. We believe hybrid integration of heterogeneous materials is the key to successful wide commercialization of such free form electronics. 


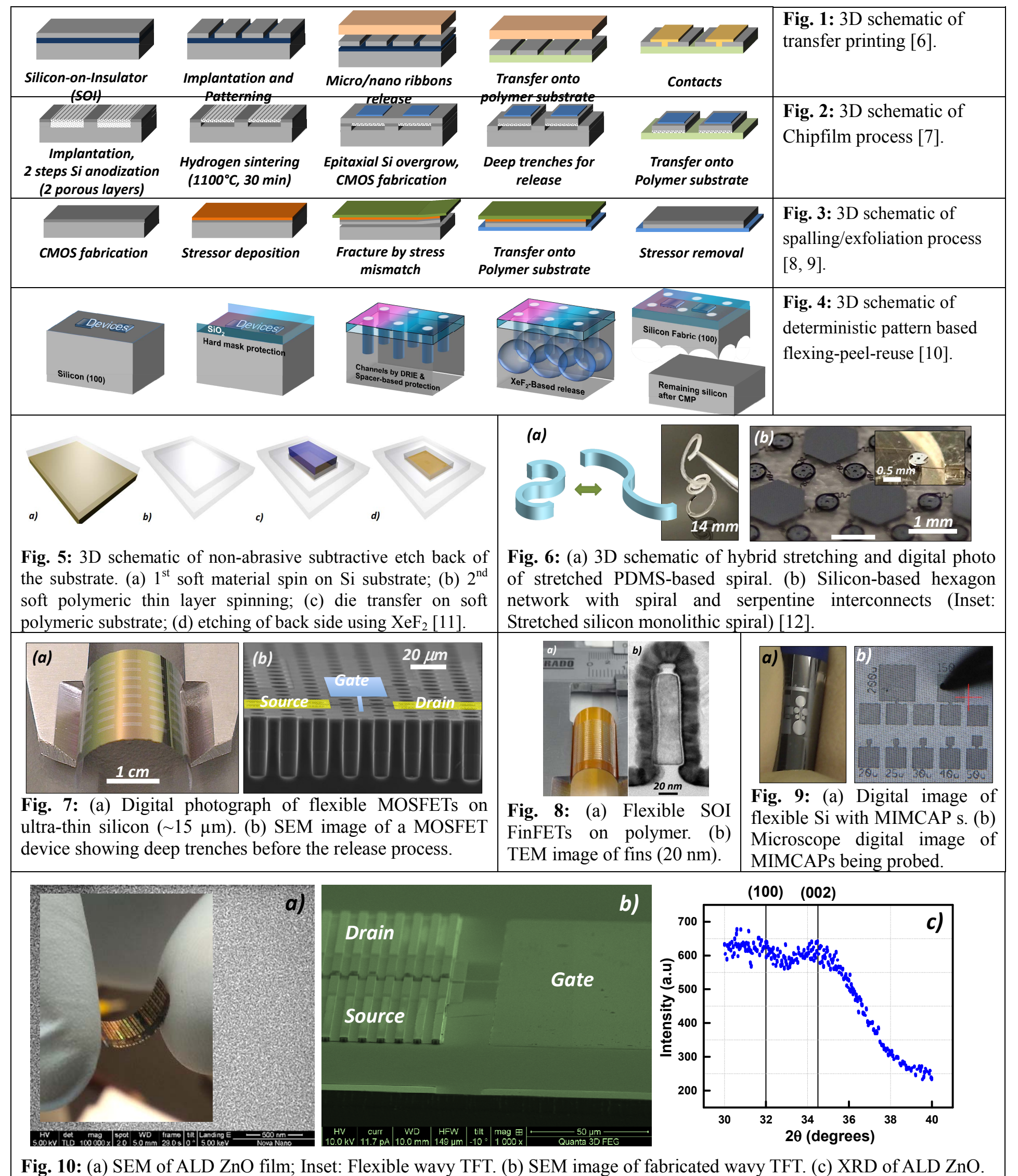




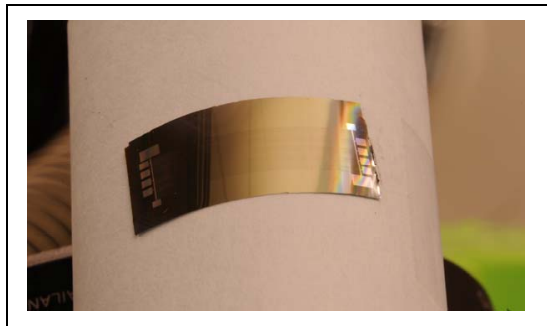

Fig. 11: Digital photograph of flexible thermoelectric generator.
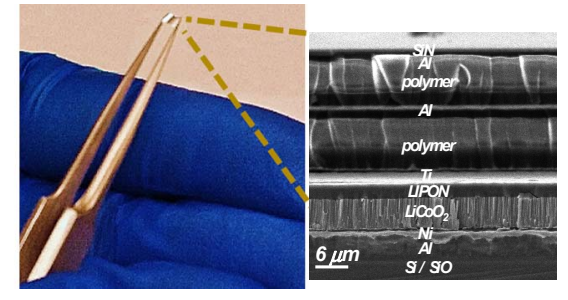

Fig. 12: Optical micrograph of flexible thin film lithium ion battery $\left(1 \mathrm{~mm}^{2}\right)$ and SEM image of the thin film stack used in the battery.
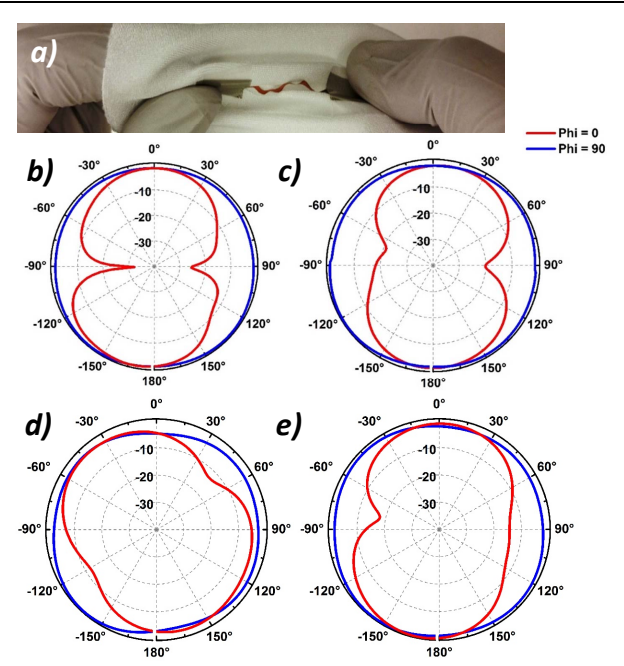

Fig. 13: (a) Optical image of the flexible and stretchable antenna on a stretchable fabric. Radiation patterns for $\mathrm{E}$ and $\mathrm{H}$ plane at the resonant frequency of $2.45 \mathrm{GHz}$ under (b) relaxed; (c) 30\% stretching; (d) bending radius $3 \mathrm{~cm}$; (e) after 1000 bending cycles.

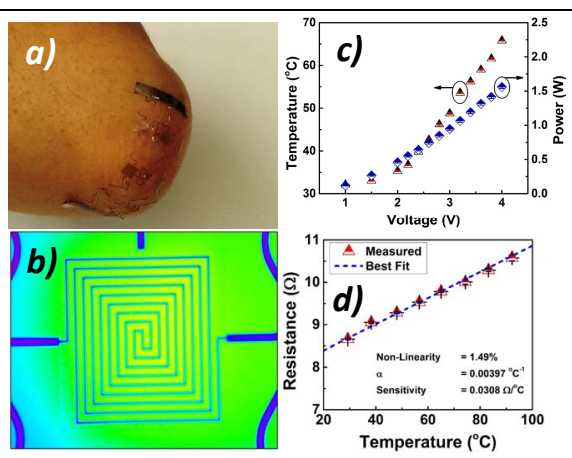

Fig. 14: (a) Optical image of the stretchable thermal patch attached to the human skin under 100\% stretching and bending radius $=5 \mathrm{~cm}$. (b) Thermal images of the metal/polymer pad on glass substrate. (c) Temperature and power consumption of the device with respect to applied voltage. (d) The device can work as a temperature sensor since the resistance of copper changes linearly with temperature.
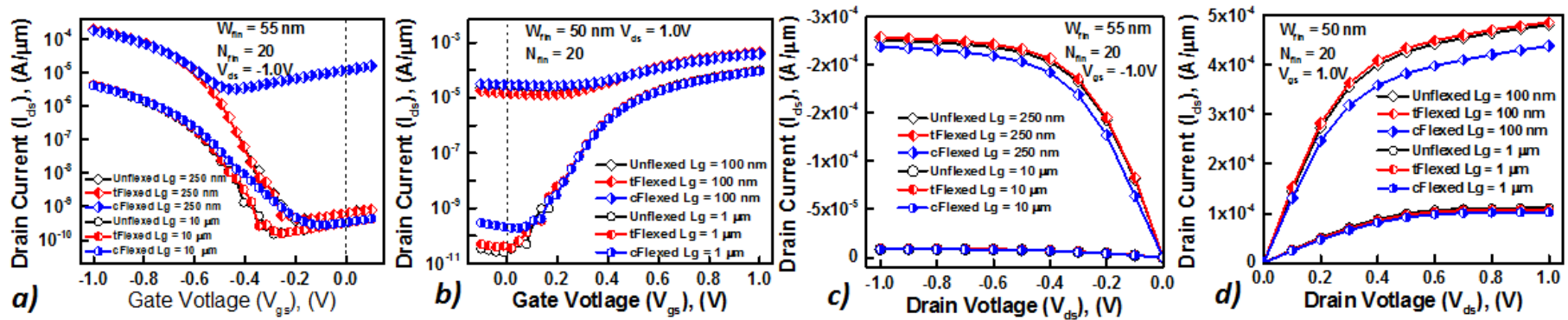

Fig. 15: Performance characteristics of a flexible SOI FinFET CMOS. Transfer curves for (a) PMOS, (b) NMOS; output plots for (c) PMOS and (d) NMOS. $\boldsymbol{t}$ and $c$ denotes bending downward and upward, respectively. $\mathrm{L}_{\mathrm{g}}=250 \mathrm{~nm}$ data from Ref. 24.

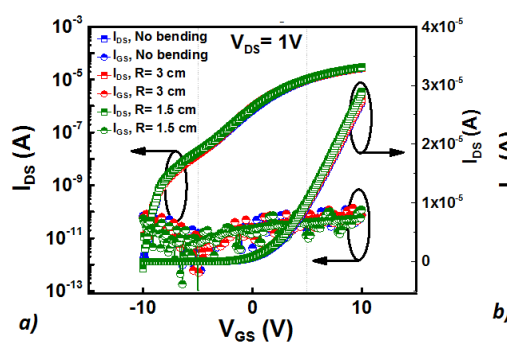

Fig. 16: Performance characteristics of wavy TFT ( $\mathrm{W}=250$ $\left.\mu \mathrm{m}, \mathrm{L}_{\mathrm{g}}=20 \mu \mathrm{m}\right)(\mathrm{a}) \mathrm{I}_{\mathrm{d}}-\mathrm{V}_{\mathrm{g}}$ and (b) $\mathrm{I}_{\mathrm{d}}-\mathrm{V}_{\mathrm{d}}$ plots (flat and bent).
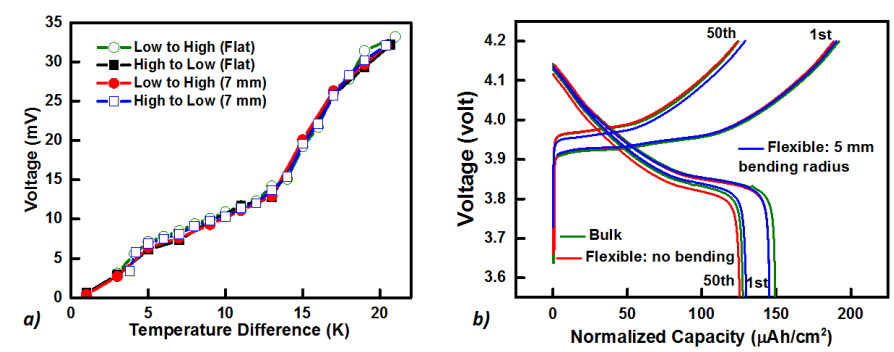

Fig. 17: (a) Performance of a flexible TEG. Charge-discharge characteristics of a flexible $\mu$ LIB.

References: [1] J. -T. Moon, IEDM 2012; [2] D. Akinwande et al. Nat. Commun. 5, 5678 (2014); [3] ITRS 2009; [4] tech@virginiasemi.com (last accessed 20 June 2015); [5] D. Hackler et al. Proc. IEEE, 103(4), 633 (2015); [6] J. He et al. Proc. IEEE 103(4), 619 (2015); [7] J. N. Burghartz, IEEE Spectrum 50(3), 38 (2013); [8] Y. Zhai et al. Nano Lett. 12(11), 5609 (2012); [9] D. Shahrjerdi et al. IEDM 2012; [10] J. P. Rojas et al. ACS Nano 8(2), 1468 (2014); [11] G. A. Torres Sevilla et al. ACS Nano 8(10), 9850 (2014); [12] J. P. Rojas et al. Appl. Phys. Lett. 105, 154101 (2014); [13] J. P. Rojas et al. Sci. Rep. 3, 2609 (2013) [14] G. A. Torres Sevilla et al. Adv. Mater. 26, 2794 (2013); [15] J. P. Rojas et al. IEEE Trans. Elect. Dev. 60(10), 3305 (2013); [16] M. T. Ghoneim et al. Adv. Elect. Mater. 1(6), (2015); [17] M. T. Ghoneim et al. Microelectronics J. 45(11), 1392 (2014); [18] G. A. Torres Sevilla et al. Small 9, 3916 (2013); [19] A. M. Hussain et al. Adv. Healthcare. Mater. 4, 665 (2015); [20] M. T. Ghoneim et al. Appl. Phys. Lett. 104, 234104 (2014); [21] M. T. Ghoneim et al. IEEE Trans. Reliab. Phys. 64(2), 579 (2014); [22] J. M. Nassar et al. pss-RRL 8(9), 794 (2014); [23] M. Nour et al. IEEE Nano 2014; [24] M. T. Ghoneim et al. DRC 2015. 